\title{
Inkjet printing of carbon black electrodes for dielectric elastomer actuators
}

Samuel Schlatter ; Samuel Rosset ; Herbert Shea

Proc. SPIE 10163, Electroactive Polymer Actuators and Devices (EAPAD) 2017, 1016311 (April 17, 2017); doi:10.1117/12.2258615

Copyright 2017 Society of Photo-Optical Instrumentation Engineers. One print or electronic copy may be made for personal use only. Systematic reproduction and distribution, duplication of any material in this paper for a fee or for commercial purposes, or modification of the content of the paper are prohibited. 


\title{
Inkjet printing of carbon black electrodes for dielectric elastomer actuators
}

\author{
Samuel Schlatter, Samuel Rosset, Herbert Shea \\ Microsystems for Space Technologies Laboratory, Ecole Polytechnique Fédérale de Lausanne, \\ Neuchâtel, Switzerland
}

\begin{abstract}
Inkjet printing is an appealing technique to print electrodes for Dielectric Elastomer Actuators (DEAs). Here we present the preparation and ink-jet printing of a carbon black electrode mixture and characterise its properties. Carbon black has been used extensively in the past because it is very compliant; however, it has a high resistance and can be very dirty to work with. In this paper we show that carbon black remains an appropriate electrode material, and when inkjet printed can be used to fabricate devices meeting today's demanding requirements. DEAs are becoming thinner to decrease actuation voltages and are shrinking in size to match the scale of the devices in the biomedical field, tuneable optics, and microfluidics. Inkjet printing addresses both of these problems. Firstly, Inkjet printing is a non-contact technique and can print on very thin freestanding membranes. Secondly, the high precision of inkjet printers makes it possible to print complex electrode geometries in the millimetre scale. We demonstrate the advantages of inkjet printing and carbon black electrodes by conducting a full characterisation of the printed electrodes. The printed carbon black electrodes have resistances as low as $13 \mathrm{k} \Omega / \square$, an elastic modulus of approximately $1 \mathrm{MPa}$, and a cyclic resistance swing which increases by $7 \%$ over 1500 cycles at $50 \%$ stretch. We also demonstrate a DEA with printed carbon black electrodes with a diametral stretch of $8.8 \%$ at an electric field of approximately $94 \mathrm{~V} / \mu \mathrm{m}$. Finally a qualitative test is conducted to show that the printed carbon black electrode is extremely hardwearing.
\end{abstract}

Keywords: dielectric elastomer actuator, inkjet, electrodes, carbon black, printing, actuator, stretchable

\section{INTRODUCTION}

Fabricating electrodes for DEAs poses many challenges. DEA electrodes must be conductive, compliant, and robust. To operate in the kilo-hertz range the equivalent electrode resistance should not exceed $1 \mathrm{M} \Omega^{1}$; the product of thickness and elastic modulus of the electrode should be less than that of the membrane ${ }^{2}$; and the electrode must maintain its conductivity and mechanical integrity over large strains $(10 \%$ to $50 \%)$ and many cycles. Finding materials with these properties is challenging because good conductors are not compliant. Another challenge is the patterning of these materials on a DEA membrane. DEAs have evolved from relatively simple geometries to more complex, integrated structures. Examples can be found in the fields of tuneable optics, biomedical applications, and microfluidics ${ }^{3}$. Generally these structures are smaller in scale (millimetre) and require new fabrication techniques to be realized.

Today, the electrodes for DEAs are fabricated by hand or with replication techniques such as stamping ${ }^{4}$, masking ${ }^{5,6}$, or transfer printing ${ }^{7}$. The replication techniques can produce devices with a good level of detail and reproducibility; however, these techniques share a common problem: they are all contact printing techniques. In the past this has not been an issue because the membranes used for DEAs were relatively thick $(20 \mu \mathrm{m}-100 \mu \mathrm{m})$. However, the trend is to fabricate devices with thinner membranes to lower actuation voltages ${ }^{8}$. Membranes which are less than $10 \mu \mathrm{m}$ thick are fragile and have a tendency to stick to objects which contact the surface of the membrane; thus becoming damaged in the process. Another problem is that contact printing techniques are not suitable for high aspect ratio structures. Integrated structures such as tuneable optics, biomedical devices and microfluidics are multi-layer, multi-material structures. As these structures are assembled and grow in height the apparent stiffness of the structure changes and the printing surface becomes increasingly uneven. This makes it difficult to reproduce the electrode on the top surface with contact printing techniques.

Inkjet printing is a non-contact printing technique which is suitable for printing DEA electrodes. DEA electrodes are large area thin films. Inkjet is well adapted to produce this type of geometry because inkjet droplets are relatively large. The diameter of the droplet on the surface is largely dependent on the nozzle diameter, thus giving droplets or lines with a width of $20 \mu \mathrm{m}-150 \mu \mathrm{m}$. In addition, the thickness of the printed electrodes can be controlled by adjusting the solid

Electroactive Polymer Actuators and Devices (EAPAD) 2017, edited by Yoseph Bar-Cohen, Proc. of SPIE Vol. 10163, 1016311 · @ 2017 SPIE · CCC code: 0277-786X/17/\$18 · doi: 10.1117/12.2258615 
content of the electrode mixture. By reducing the solid content of the electrode mixture it is possible to reduce the thickness of the electrode layer. Furthermore, inkjet is an established technology. Multi-jet systems are available for fast parallel printing of centimetre scale devices, multi head systems are available for printing multi-material structures, and a large research database exists of materials and techniques. Inkjet is therefore well suited for printing today's millimetre scale DEA devices.

A number of studies have used inkjet printing to produce stretchable conductors on PDMS membranes. The most conductive of which are metal electrodes. Chung et al. ${ }^{9}$ has demonstrated that a silver electrode printed on a wavey PDMS substrate produces conductors with a sheet resistance of $0.44 \Omega / \square$. The absolute resistance increased by a factor 2.75 when stretched to $10 \%$ over 1000 cycles. Robinson et al. ${ }^{10}$ has shown that highly conductive lines can be produced by printing silver ink onto a micro-structured silicone substrate. The print lines were shown to have a resistance of $140 \Omega$ for a $5 \mathrm{~mm}$ long conductor with a width of $210 \mu \mathrm{m}$, a sheet resistance of approximately $5.9 \Omega / \square$. These techniques produce excellent conductors for low voltage electronics but are not optimal for DEAs. Metal electrodes are stiff due to the high elastic modulus of metals, the PDMS substrate requires micro-structuring, and the printed conductors need to be sintered after printing. Carbon based materials are better suited for DEA electrodes. Baechler et al. ${ }^{11}$ demonstrated inkjet printed Multiwall Carbon Nanotube (MWCNT) electrodes on PDMS, with a sheet resistance of $6.4 \mathrm{k} \Omega / \square$, increasing by a factor of 20 at $50 \%$ strain. A functional DEA was also demonstrated with a biaxial strain of $3 \%$.

In this paper, carbon black is used as an electrode material. Carbon black has been used extensively to produce DEA electrodes because it is sufficiently conductive and has a very low impact on the effective stiffness of a DEA. However, this is only true when the carbon black is in powder form or combined with a silicone oil to form conductive grease. The main drawback of carbon black in these forms is that they do not adhere well to PDMS membranes and thus do not form very robust electrodes. We believe that this is only the case because the material is applied manually and in excess. We therefore reinvestigate the usability and performance of carbon black as an electrode when printed. First, the recipe and the printing parameters required to print the material are documented. Second, the electrode is characterised for conductivity, stiffness, and cyclic conductivity; these are the 3 main characteristics which should be considered when comparing DEA electrodes. Lastly, the methods presented in this paper are used to print the electrodes of a functional DEA.

\section{ELECTRODE MIXTURE PREPARATION}

The electrode mixture documented in this proceeding is a carbon black based, binder-less electrode. The electrode consists of three materials: carbon black, a dispersant, and a solvent. These are combined in a three step process to produce a printable electrode mixture. Step 1) the carbon black (Ketjenblack EC-300J, AkzoNobel) and the dispersant (Belsil®) SPG 128 VP, Wacker Chemie AG) are combined in a ratio of $1 \mathrm{~g}: 12 \mathrm{~g}$. The ingredients are milled with six 12 $\mathrm{mm}$ ball bearings in a Retsch PM200 ball mill at 650 RPM for 14 min. Step 2) the carbon-dispersant mixture and a silicone solvent (OS-2, Dow Corning ${ }^{\circledR}$ ) are combined in a conical test tube in a ratio of $4 \mathrm{ml}: 14 \mathrm{ml}$. The mixture is then sonicated for $5 \mathrm{~min}$ to disperse the carbon-dispersant. Step 3) the electrode mixture is filtered by centrifugation to avoid clogging of the printer nozzle. The mixture is transferred into $1.5 \mathrm{ml}$ Eppendorf tubes and centrifuged at $13 \mathrm{kRPM}$ for 1 min. Following centrifugation $0.75 \mathrm{ml}$ of the mixture is removed from the top of the Eppendorf tubes and transferred to a clean printer vial.

\section{PRINTING OF ELECTRODE MIXTURE WITH JETLAB4}

The jetlab ${ }^{\circledR} 4$ (MicroFab) is a Drop-on-Demand table-top printing platform. It consists of a XYZ stage, cameras to monitor printing, and a dispensing device. A low temperature, piezo-electric dispensing device with a $50 \mu \mathrm{m}$ orifice was used for the following experiments. To print with this device, it is necessary to determine the jetting parameters which lead to stable jetting, and the printing parameters which produce a continuous electrode.

The jetting parameters define the shape of the electrical waveform sent to the piezo-electric dispensing device. The simplest waveform, a trapezoidal waveform, did not produce stable jetting and hence a bipolar trapezoidal waveform was used instead. A symmetrical waveform was chosen as a starting point and the length of the waveform was minimised to reduce droplet size. Following this the amplitude was adjusted to promote stable droplet generation. The jetting parameters which led to stable droplet generation are listed in Table 1. 
Table 1. Jetting parameters for jetlab ${ }^{\circledR} 4$ printer with a $50 \mu \mathrm{m}$ piezo-electric dispensing device. These parameters produce a trapezoidal waveform, where the first pulse is approximately half the amplitude of the second. The frequency of jetting was set to $300 \mathrm{~Hz}$.

\begin{tabular}{|c|c|c|c|c|c|c|c|}
\hline Rise time 1 & Dwell time & Fall time & Echo time & Rise time 2 & Idle voltage & Dwell voltage & Echo voltage \\
\hline $2 \mu \mathrm{s}$ & $3 \mu \mathrm{s}$ & $6 \mu \mathrm{s}$ & $3 \mu \mathrm{s}$ & $4 \mu \mathrm{s}$ & $0 \mathrm{~V}$ & $40 \mathrm{~V}$ & $-85 \mathrm{~V}$ \\
\hline
\end{tabular}

The printing parameters define the placement of the drops on the substrate. The most critical printing parameter to print an electrode is the droplet spacing. The individual drops must overlap to form a continuous electrode. To determine a suitable droplet spacing an array of dots was printed on a silicone membrane (Elastosil ${ }^{\circledR}$ Film 2030 250/20, Wacker Chemie AG) and the diameter of the drop was measured with a digital microscope (KH-8700, HiROX). The droplets have an approximate diameter of $125 \mu \mathrm{m}$ and hence a spacing of $50 \mu \mathrm{m}$ was chosen. A second array was printed to confirm that this droplet spacing leads to overlap and hence a continuous electrode (Figure 1).
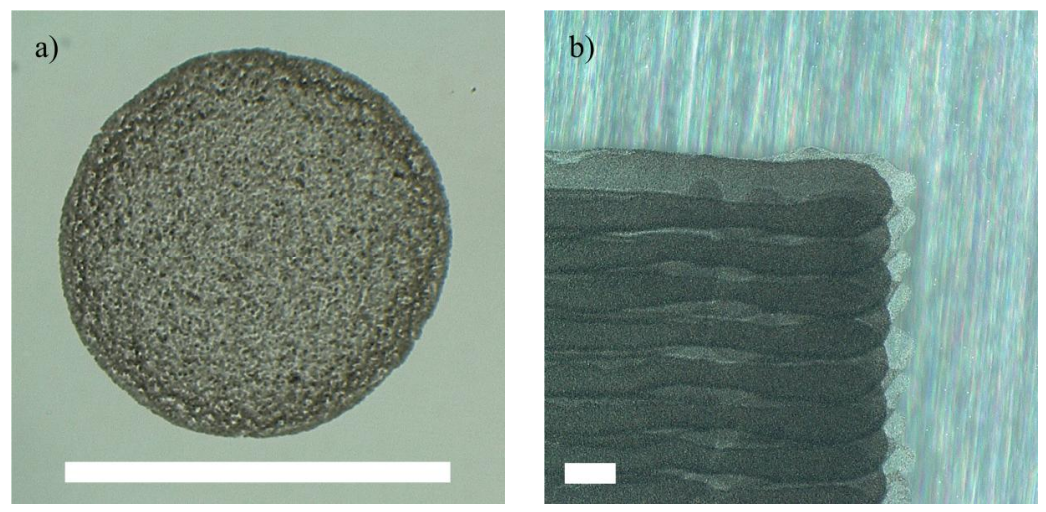

Figure 1. a) Bright field digital microscope image of a droplet on an Elastosil ${ }^{\circledR}$ membrane. The length of the scale bar is $125 \mu \mathrm{m}$. b) Dark field image of an array printed with a horizontal and vertical droplet spacing of $50 \mu \mathrm{m}$. The chromatic aberration is due to the surface texture on the backing of the Elastosil@ membrane. The scale bar is $125 \mathrm{um}$.

\section{CHARACTERISATION OF THE PRINTED ELECTRODES}

In this section we present the methods and results of three experiments to measure the conductivity, stiffness, and cyclic conductivity of the printed electrodes.

\subsection{Conductivity of printed electrodes}

The following experiment was conducted to determine the resistance of a printed electrode strip when stretched uniaxially. Four samples were prepared which differ by the number of printed layers and the printing direction. The samples were fabricated by laser cutting a sheet of Elastosil ${ }^{\circledR}$ Film 2030 250/20 (Wacker Chemie AG) into a rectangular shape - with the backing intact. The laser cut films were then placed on to the printer table and a $5 \mathrm{~mm} \times 55 \mathrm{~mm}$ electrode strip was printed on to the surface of this film. A digital microscope image of the printed electrodes is shown in Figure 2. The parallel samples were printed with the printing direction parallel to the electrode strip, the perpendicular sample was printed with the printing direction perpendicular to the electrode strip, and the crosshatched sample is a combination of the two. The thickness of the printed electrodes was measured with a Wyko NT1100 optical profiler. The electrode thickness of the samples was measured to be $0.38 \mu \mathrm{m}$ (sample 1), $1.08 \mu \mathrm{m}$ (sample 2), $0.52 \mu \mathrm{m}$ (sample 3), and $1.00 \mu \mathrm{m}$ (sample4). 


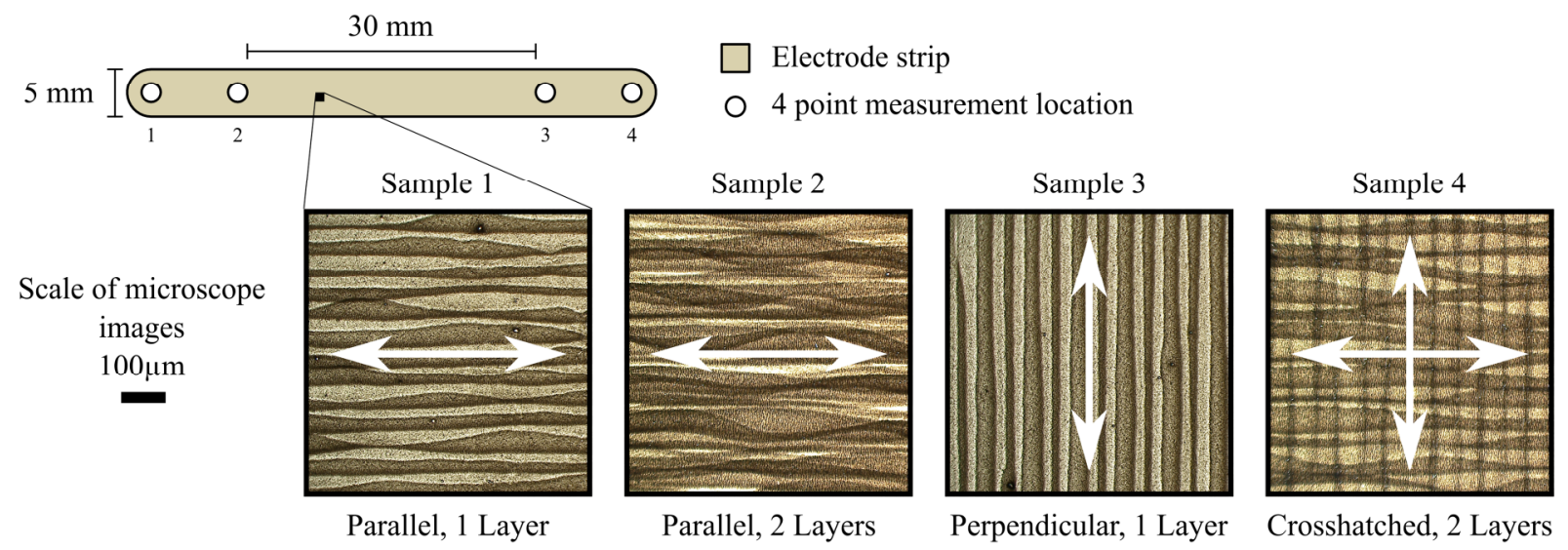

Figure 2. The figure shows microscope images of the samples used for the conductivity measurements; the images were taken at arbitrary locations on the electrode strip. The printing direction is indicated with white arrows on the microscope images. The diagram shows the dimensions of the electrode strip and the location of the four points for the four point resistance measurement. A current was applied to points $1 \& 4$, and the voltage was measured across points $2 \& 3$. The region between points $2 \& 3$ was stretched from $30 \mathrm{~mm}$ to $45 \mathrm{~mm}$.

The samples were then placed on a linear stage and stretched with a triangular displacement profile. The stage was programmed to move $15 \mathrm{~mm}$, stretching the section of the electrode strip under test from $30 \mathrm{~mm}$ to $45 \mathrm{~mm}$ (stretch of 1.5). The samples were stretched at a low speed of $1 \mathrm{~mm} / \mathrm{s}$ to avoid rate dependent resistance effects. The test was repeated 15 times and the resistance was measured with a four point measurement method. The resistance of the newly fabricated samples was observed to change significantly in the first five cycles, settling to a stable value after approximately 5 cycles. The $15^{\text {th }}$ cycle of each sample is shown in Figure 3.
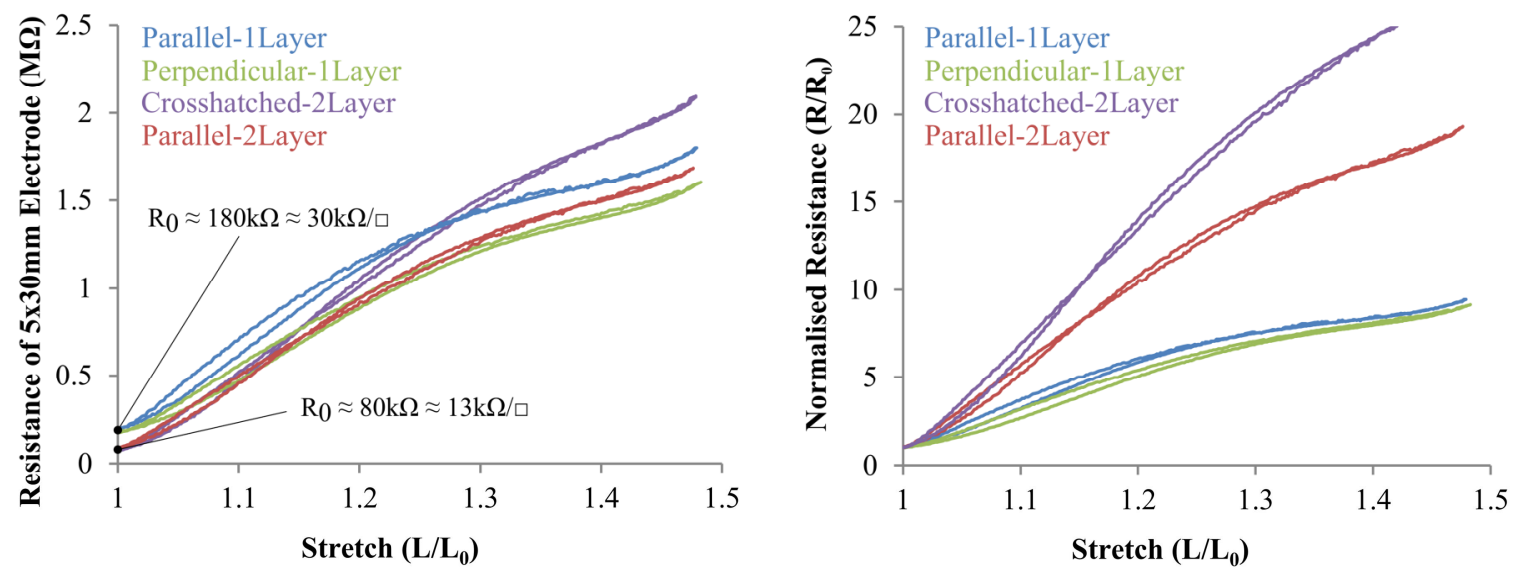

Figure 3. (left) Absolute resistance of printed electrode on the 15th cycle when stretched by $50 \%$ (from $30 \mathrm{~mm}$ to $45 \mathrm{~mm}$ ). The sheet resistance is indicated for the 1 Layer electrodes and the 2 Layer electrodes in the unstretched state. (right) The normalized resistance of the data shown on the left.

\subsection{Modulus of elasticity of printed electrode}

To determine the elastic modulus of the electrode it was printed on a thin membrane of soft PDMS (MED-4086, NuSil) with a shore hardness 000 of 55 , or an elastic modulus of $\sim 46 \mathrm{kPa}$; the impact of the electrode on a soft membrane is more pronounced and hence more easily measured. The samples were fabricated by blade casting PDMS onto a substrate coated with a water soluble sacrificial layer ${ }^{4}$, adding a frame to hold the membrane, and removing the substrate prior to testing (Figure 4). The PDMS was prepared by first mixing $5 \mathrm{~g}$ of part A with $5 \mathrm{~g}$ of part $\mathrm{B}$ in a planetary mixer. Following this, $10 \mathrm{~g}$ of silicone solvent (OS-2, Dow Corning ${ }^{\circledR}$ ) were added, and the mixture was again mixed with the 
planetary mixer. The PDMS mixture was blade cast at a height of $40 \mu \mathrm{m}$ and the membrane was cured at $150^{\circ} \mathrm{C}$ for 45 min. Once cured, the membrane was laser cut into $2 \mathrm{~cm}$ squares and a frame was added. Following this the electrode was printed on the surface of the membrane with the substrate still intact. Three samples were fabricated: 1) a bare membrane as a control, 2) a membrane with a single layer electrode, and 3) a membrane with a two layer electrode. The full surface of the membrane was covered by printing an electrode slightly larger than the section of the membrane being tested. With the electrode in place, the samples were placed into a bath of boiling water to dissolve the sacrificial layer and release the PET substrate; Belsil 128, the dispersant used in the electrode mixture, is partially soluble in water, however, no carbon was visible in the water bath after releasing the PET substrate. After releasing the membrane the samples were removed from the water bath, and the excess water was evaporated by placing the samples in an oven at $80^{\circ} \mathrm{C}$ for $15 \mathrm{~min}$.

The samples were tested on a setup consisting of a linear motor and a load cell. The samples were attached with adhesive to the moving parts of the setup and the locking strips on the sides of the samples were removed (Figure 4). The samples were then subjected to a triangular displacement profile with an amplitude of $400 \mu \mathrm{m}$. The results of the tests are shown in Figure 4.
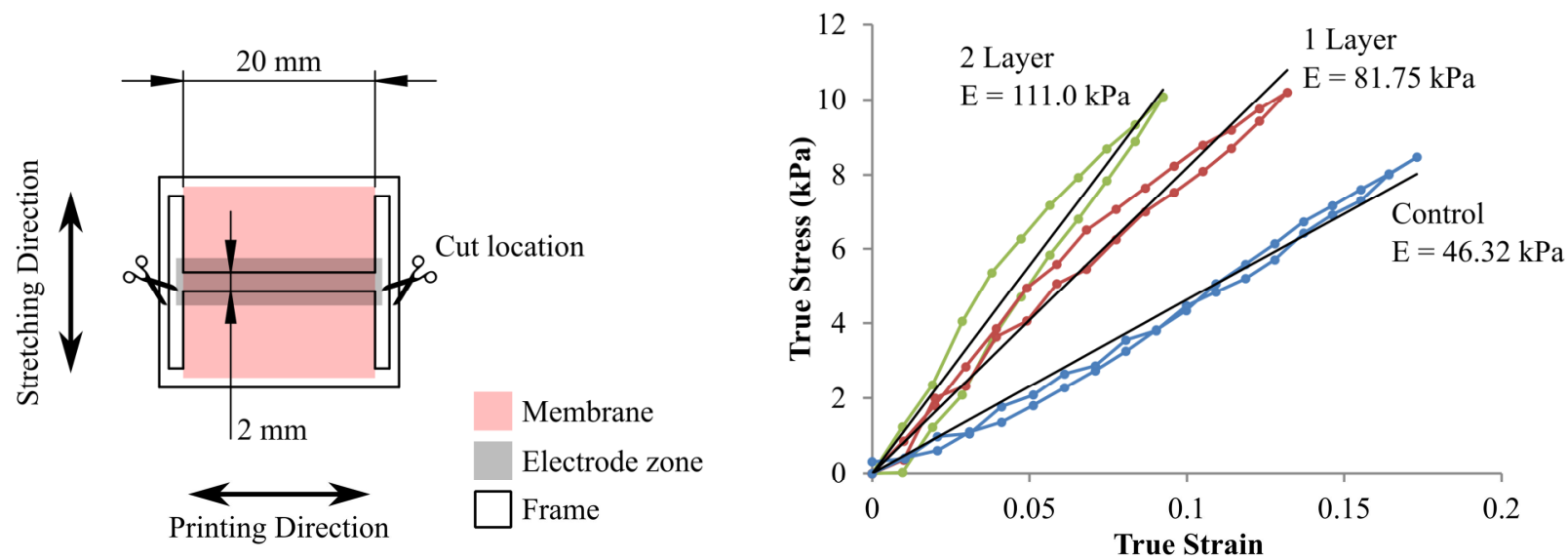

Figure 4. (left) A diagram of the samples used to measure the elastic modulus of the printed electrode. The samples consist of two parts: a square membrane on a PET substrate (coated with a water soluble material), and a PET frame with a silicone adhesive. The electrode was printed on to the membrane surface perpendicular to the stretching direction. (right) The true stress-strain curves of the three tested samples. A linear correlation was fitted to the curves to determine the elastic modulus of each sample.

The elastic moduli in Figure 4 are the elastic moduli of multilayer structures. To determine the elastic modulus of the electrode alone the Voigt model was used. The Voigt model simplifies to equation 1 when the width and the length of the two materials are the same ${ }^{1}$.

$$
\begin{gathered}
E_{\text {effective }}=\frac{t_{\text {membrane }}}{t_{\text {total }}} E_{\text {membrane }}+\frac{t_{\text {electrode }}}{t_{\text {total }}} E_{\text {electrode }} \\
\text { where } E=\text { elastic modulus, } t=\text { thickness }
\end{gathered}
$$

The thickness of the membrane and the electrodes was measured experimentally. The thickness of the membrane was measured with a white light interferometer, giving a thickness of $11.95 \mu \mathrm{m}$. The thickness of the electrodes was obtained by measuring the samples from section 4.1. The step at the edge of the electrodes was measured with a Wyko NT1100 optical profiler. The effective elastic modulus of the composite and that of the membrane are obtained from the tensile test presented above. The results of these measurements are shown in Table 2, along with an estimate of the elastic modulus of the electrode, calculated using Equation 1.

Table 2. A table showing the thickness and elastic modulus of respective layers.

\begin{tabular}{|l|c|c|c|c|c|c|}
\cline { 2 - 7 } \multicolumn{1}{c|}{} & $\mathrm{t}_{\text {membrane }}(\mu \mathrm{m})$ & $\mathrm{t}_{\text {electrode }}(\mu \mathrm{m})$ & $\mathrm{t}_{\text {total }}(\mu \mathrm{m})$ & $\mathrm{E}_{\text {effective }}(\mathrm{kPa})$ & $\mathrm{E}_{\text {membrane }}(\mathrm{kPa})$ & $\mathrm{E}_{\text {electrode }}(\mathrm{kPa})$ \\
\hline 1 Layer Electrode & 11.95 & 0.38 & 12.33 & 81.75 & 46.32 & 1196 \\
\hline 2 Layer Electrode & 11.95 & 1.10 & 13.05 & 111.0 & 46.32 & 814.1 \\
\hline
\end{tabular}




\subsection{Cyclic conductivity of printed electrode}

In this section the conductivity of the printed electrode is observed over 1500 stretch cycles at $50 \%$ uniaxial strain. The sample used for the cyclic tests was prepared according to the methods presented in section 4.1. One sample was produced with a parallel printed single layer electrode. To stretch the sample, a crank mechanism was used. The setup consists of a DC motor, a crank, and a slide which converts rotational motion into sinusoidal linear motion. The motor was set to rotate at a fixed speed of 23 RPM and the cycles were counted with a counter. The resistance was measured with a four point measurement method every 100 cycles. The maximum resistance, which corresponds to the resistance in the stretched state; and the minimum resistance, which corresponds to the un-stretched state, have been plotted in Figure 5. A normalised plot is also given to show more clearly the evolution of the maximum and minimum resistance.
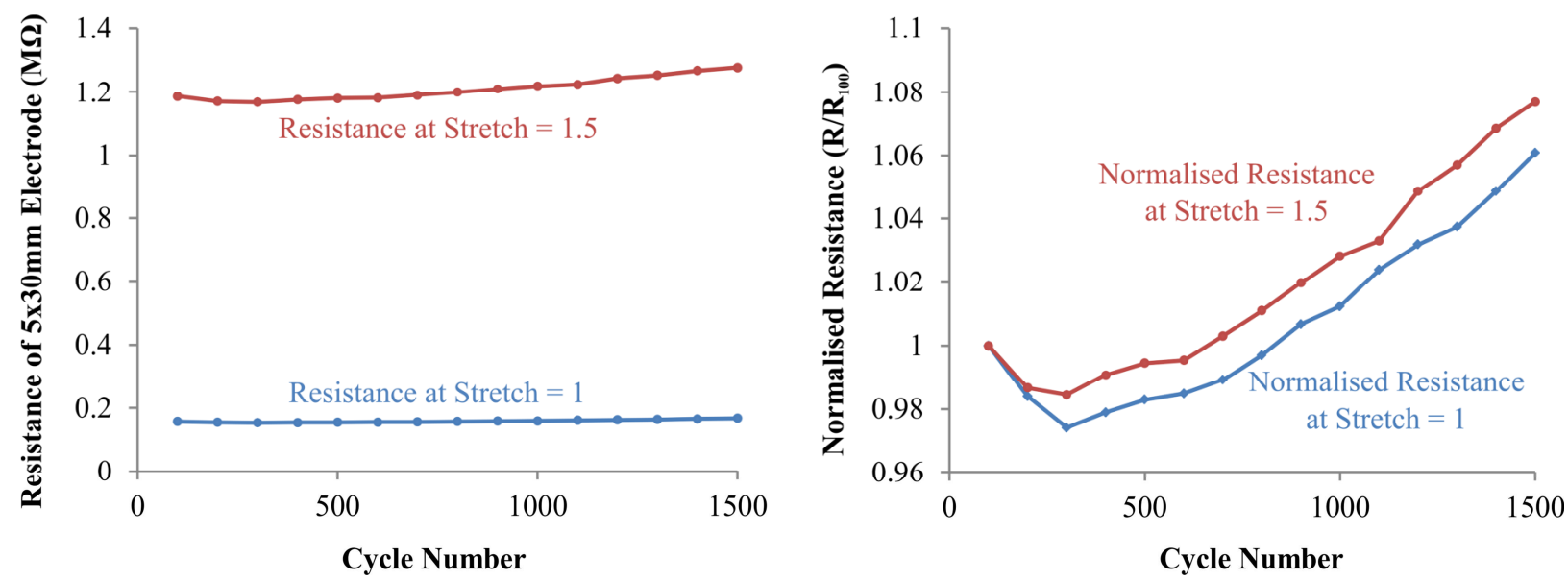

Figure 5. (left) A plot showing the maximum and minimum resistance of a parallel printed single layer electrode when stretched to $50 \%$ over 1500 cycles. The resistance is for an electrode strip with a width of $5 \mathrm{~mm}$ and a length of $30 \mathrm{~mm}$. (right) Normalised resistance plot showing the evolution of the maximum and minimum resistance. Note that the maximum resistance is six times the minimum resistance at cycle 100 .

\section{DEA WITH INKJET PRINTED ELECTRODES}
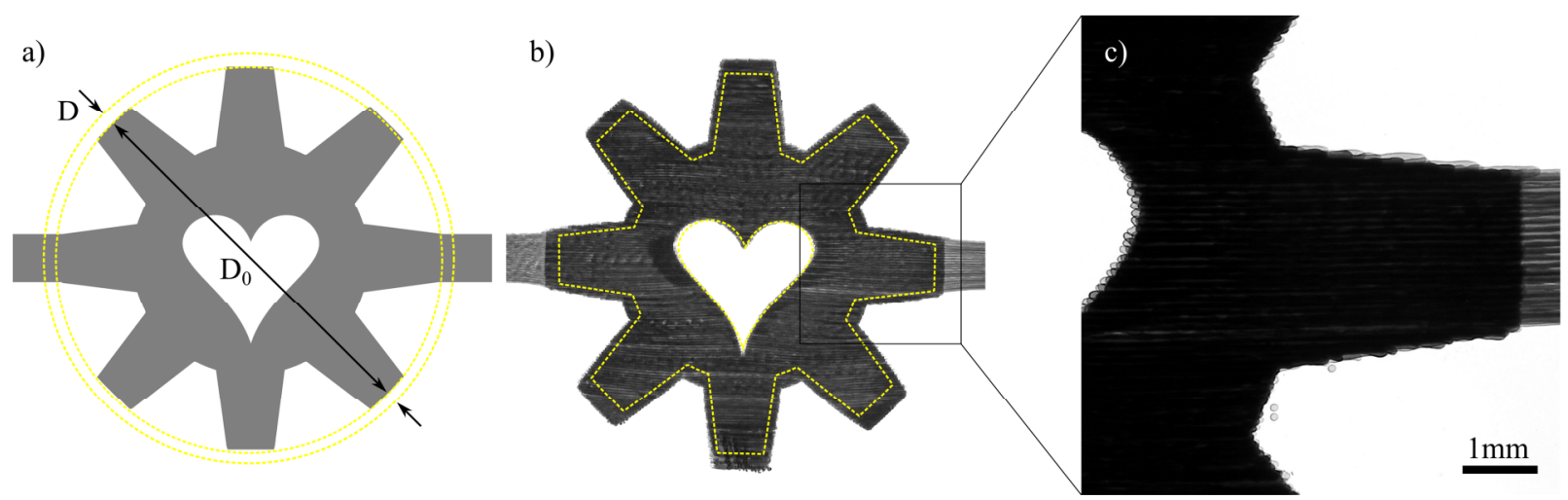

Figure 6. (a) The shape of the printed electrodes indicating the diameter of the cog at rest $\left(\mathrm{D}_{0}\right)$ and when actuated (D). At rest the $\operatorname{cog}$ has a diameter of approximately $17 \mathrm{~mm}$. (b) Photograph of DEA with ink-jet printed electrodes when actuated at approximately $94 \mathrm{~V} / \mu \mathrm{m}$. The outline of the actuator at rest is super imposed on the photograph (c) A close up of the ink-jet printed electrodes.

In this section a functional DEA is fabricated with the electrode mixture and printing parameters introduced in this paper. A beating heart actuator was chosen to illustrate the printing of a relatively complex shape. The actuator was prepared according to our standard actuator fabrication protocol ${ }^{4}$, where a beating heart actuator is fabricated using pad printing. 
Steps $1 \& 2$ of the protocol were followed to prepare the prestretched membranes. Two membranes were prepared: sample 1 with a thickness of $24.55 \mu \mathrm{m}$ and a prestretch of 1.22 , and sample 2 with a thickness of $21.48 \mu \mathrm{m}$ and a prestretch of 1.28. Step 3 of the protocol, Patterning Compliant Electrodes by Pad Printing was replaced by inkjet printing to pattern the electrodes. The electrodes were printed on each side of the silicone membrane in a single printing pass with the power lines parallel to the printing direction (Figure 6). The connections to the electrodes were made according to step 4 of the protocol.

A simple setup was used to characterise the expansion of the printed actuator. The setup consisted of a USB microscope, a lightbox to improve the contrast of the images, and a high voltage DC power source. The actuator was connected to the DC power source and the voltage was increased in a nonlinear fashion to produce a linear change in stretch, i.e. the voltage was increased more rapidly at low voltages and more slowly at high voltages. A photo was taken at each voltage setpoint and the photo sequence was imported into NI Vision Assistant 2013 to measure the outside diameter of the cog (Figure 6). The diameter was then used to calculate the diametral stretch $\left(\mathrm{D} / \mathrm{D}_{0}\right)$ and the electric field at each voltage setpoint. The results are compared with pad printed actuators developed according to the Jove protocol ${ }^{4}$ with electrodes consisting of a carbon black and silicone elastomer composite (Figure 7).

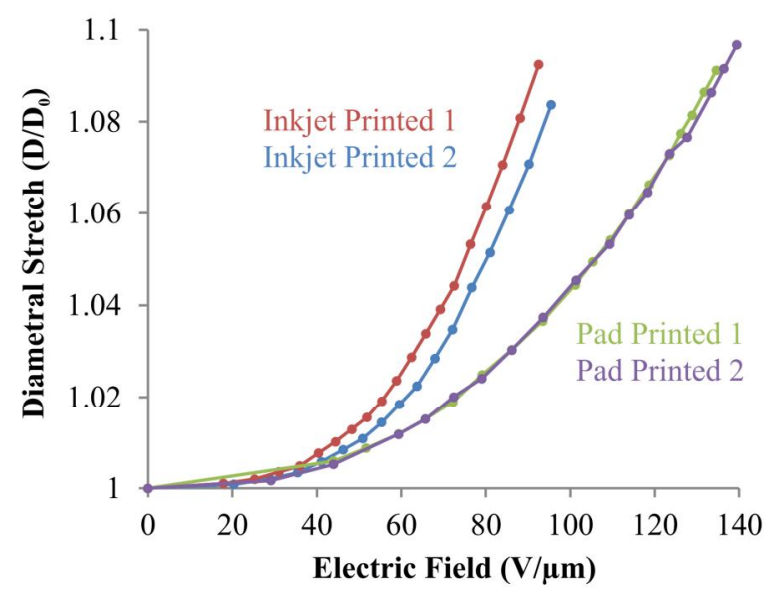

Figure 7. A comparison plot showing the diametral stretch of four actuators in response to an electric field. The pad printed actuators were developed according to the Jove protocol ${ }^{4}$ with electrodes consisting of a carbon black and silicone elastomer composite. The inkjet printed actuators were developed with the methods presented in this paper consisting of carbon black and a dispersant.

\section{DISCUSSION}

The conductivity of the printed electrodes was measured in Section 4.1. The resistance of the samples is highly dependent on the number of layers. In the un-stretched state the single layer electrodes (approx. $0.5 \mu \mathrm{m}$ thick) have a sheet resistance of $30 \mathrm{k} \Omega / \square$ and the electrodes with two layer electrodes (approx. $1 \mu \mathrm{m}$ thick) have a sheet resistance of $13 \mathrm{k} \Omega / \square$. In the stretched state we see that the two layer electrodes undergo a larger change in resistance than the single layer electrodes. We can therefore conclude that 2 layer electrodes would be more suitable for low strain actuators which require a high conductivity, and single layer electrodes may be more suitable for electrodes subjected to strains greater than $50 \%$. This is in line with the findings of Baechler et al. ${ }^{11}$ which has also observed that thick film electrodes undergo a larger resistance change.

The elastic modulus of the printed electrodes was investigated in Section 4.2. The experiment shows that the elastic modulus of the membrane-electrode structure increases with the number of electrode layers. The elastic modulus of the electrode was calculated to be $1196 \mathrm{kPa}$ for the 1 layer electrode sample and $814 \mathrm{kPa}$ for the 2-layer electrode sample, giving an average modulus of $1005 \mathrm{kPa}$. These numbers are highly dependent on the thickness of the electrode layer. For example, if a thickness of $0.5 \mu \mathrm{m}$ is used for the 1 layer electrode and $1.0 \mu \mathrm{m}$ for the 2-layer electrode the modulus changes to $928 \mathrm{kPa}$ and $884 \mathrm{kPa}$. These values are in better agreement suggesting that the thicknesses used to calculate the elastic modulus do not match the thickness of the samples. Nevertheless, it can be concluded that the electrode material presented in this proceeding has an elastic modulus comparable to that of Sylgard 186 of approximately $1 \mathrm{MPa}^{1}$. 
The cyclic performance of the electrode was investigated in Section 4.3. The resistance was measured to vary between a minimum of $0.16 \mathrm{M} \Omega$ and a maximum of $1.19 \mathrm{M} \Omega$ in the first 100 cycles at $50 \%$ stretch. After 1500 cycles the electrode resistance increased by approximately $7 \%$ and varied between a minimum of $0.17 \mathrm{M} \Omega$ and maximum of $1.28 \mathrm{M} \Omega$. The experiment shows that the electrodes do not degrade rapidly and can be used over thousands of cycles.

The DEA with inkjet printed electrodes was produced to determine how the electrode characteristics (conductivity and elastic modulus) combine to make a functioning actuator. The stretch versus electric field plot shows that the actuator with inkjet printed binder-less electrodes reaches the same diametral stretch at a lower electric field than the pad printed electrodes. This is in part due to the lower elastic modulus of the binder-less electrodes. However, it is not possible to draw concrete conclusions from the given data because a different batch of Sylgard ${ }^{\circledR} 186$ (Dow Corning) was used to fabricate the actuators. It is possible that the difference between the two datasets is more strongly affected by the differences of the silicone membrane than the differences of the electrodes. Nevertheless, the printed electrodes were shown to produce a functional actuator. Furthermore the actuator shows that the method presented here can be used to pattern complex electrode geometries on a freestanding membranes.

Finally, to show that the electrodes are hard wearing a qualitative touch test was conducted. Two samples, 1 layer and 2 layer, were used as specimens to determine the adhesion of the printed electrode on the membrane. A finger was pushed onto the electrode and the finger and electrode were examined. In both cases a light imprint was left on the electrode, but the electrode remains largely intact. This shows that the electrode is significantly more robust than carbon powder and carbon grease electrodes.

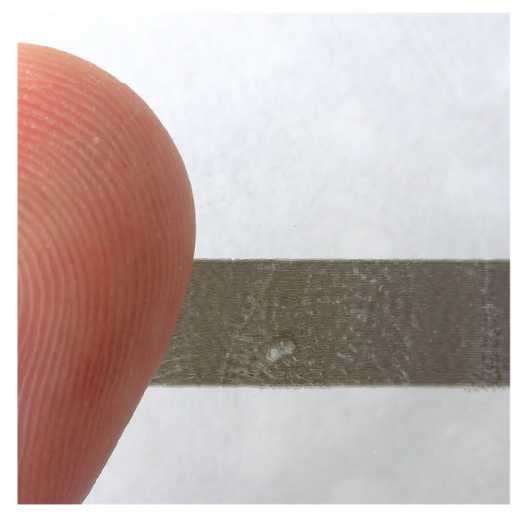

a) Parallel printed, 1 layer electrode

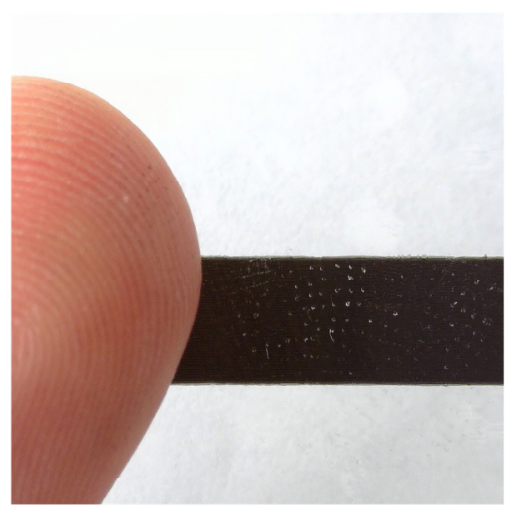

b) Parallel printed, 2 layer electrode

Figure 8. Two photographs of printed electrodes (5mm wide strip) after a qualitative touch test. In both cases very little carbon black was transferred to the finger.

\section{CONCLUSIONS}

A new carbon black based printed electrode has been presented. The printed electrode was characterised to enable comparison with other printed electrodes. The electrode has a sheet resistance between $13 \mathrm{k} \Omega / \square$ and $30 \mathrm{k} \Omega / \square$ in the unstretched state. When stretched to $50 \%$ the absolute resistance of the 1 layer $(\sim 0.5 \mu \mathrm{m}$ thick $)$ and 2 layer $(\sim 1 \mu \mathrm{m}$ thick $)$ electrodes increased by a factor of 9 and 24, respectively. The printed electrode has an elastic modulus of approximately $1 \mathrm{MPa}$. The cyclic tests showed that the maximum $(1.2 \mathrm{M} \Omega)$ and minimum $(0.16 \mathrm{M} \Omega)$ resistance of a single layer printed electrode increased by approximately $7 \%$ over 1500 cycles at a stretch of $50 \%$. Finally, a DEA was fabricated with printed electrodes, exhibiting a diametral stretch of approximately $8.8 \%$ at an electric field of approximately $94 \mathrm{~V} / \mu \mathrm{m}$.

The extensive testing shows that inkjet printing of carbon electrodes is suitable for today's DEAs. The printed electrode has a conductivity which is in a range suitable for DEAs, the product of thickness and elastic modulus is less than that of the membrane (when using membranes thicker than $1 \mu \mathrm{m}$ with an elastic modulus greater than $1 \mathrm{MPa}$ ), and the electrode does not degrade significantly at large strains over many cycles. We have demonstrated that inkjet is a suitable technique for printing electrodes on supported as well as freestanding membranes, and can be used to print on thin membranes which would otherwise be damaged by contact printing techniques. Overall, the methods presented here can be combined with other printed materials to produce complex integrated structures. 


\section{ACKNOWLEDGMENTS}

We thank Peter van der Wal for advice on dispersions, Francesca Sorba for the help with the measurements of elastic modulus, Christine de Saint-Aubin and David McCoul for sharing their experience and tips regarding the jetlab 4 system, and the members of the soft transducers group at EPFL-LMTS for their help and support.

This project has received funding from the European Union's Horizon 2020 research and innovation programme under the Marie Skłodowska-Curie grant agreement No 641822 - MICACT.

\section{REFERENCES}

[1] Rosset, S.., Shea, H. R., "Flexible and stretchable electrodes for dielectric elastomer actuators," Applied Physics A 110(2), 281-307, Springer (2013).

[2] McCoul, D., Hu, W., Gao, M., Mehta, V.., Pei, Q., "Recent Advances in Stretchable and Transparent Electronic Materials," Advanced Electronic Materials, Wiley Online Library (2016).

[3] Rosset, S.., Shea, H. R., "Small, fast, and tough: Shrinking down integrated elastomer transducers," Applied Physics Reviews 3(3), 031105, AIP Publishing (2016).

[4] Rosset, S., Araromi, O. A., Schlatter, S.., Shea, H. R., "Fabrication process of silicone-based dielectric elastomer actuators," Journal of visualized experiments: JoVE(108), MyJoVE Corporation (2016).

[5] Fasolt, B., Hodgins, M.., Seelecke, S., "Characterization of screen-printed electrodes for Dieletric Elastomer (DE) membranes: influence of screen dimensions and electrode thickness on actuator performance," SPIE Smart Structures and Materials+ Nondestructive Evaluation and Health Monitoring, 97983E-97983E (2016).

[6] Rizzello, G., Naso, D., York, A.., Seelecke, S., "Closed loop control of dielectric elastomer actuators based on self-sensing displacement feedback," Smart Materials and Structures 25(3), 035034, IOP Publishing (2016).

[7] Araromi, O. A., Rosset, S.., Shea, H. R., "High-resolution, large-area fabrication of compliant electrodes via laser ablation for robust, stretchable dielectric elastomer actuators and sensors," ACS applied materials $\backslash \&$ interfaces 7(32), 18046-18053, ACS Publications (2015).

[8] Poulin, A., Rosset, S.., Shea, H. R., "Printing low-voltage dielectric elastomer actuators," Applied Physics Letters 107(24), 244104, AIP Publishing (2015).

[9] Chung, S., Lee, J., Song, H., Kim, S., Jeong, J.., Hong, Y., "Inkjet-printed stretchable silver electrode on wave structured elastomeric substrate,” Applied Physics Letters 98(15), 153110, AIP Publishing (2011).

[10] Robinson, A. P., Minev, I., Graz, I. M.., Lacour, S. P., "Microstructured silicone substrate for printable and stretchable metallic films," Langmuir 27(8), 4279-4284, ACS Publications (2011).

[11] Baechler, C., Gardin, S., Abuhimd, H.., Kovacs, G., "Inkjet printed multiwall carbon nanotube electrodes for dielectric elastomer actuators," Smart Materials and Structures 25(5), 055009, IOP Publishing (2016). 